\title{
Dynamic compression of lightweight interpenetrating phase composite (IPC) foams: Measurements and modeling
}

\author{
C. Periasamy1 and H. Tippur ${ }^{1, \mathrm{a}}$ \\ ${ }^{1}$ Department of Mechanical Engineering, Auburn University, Auburn, Alabama, USA.
}

In recent years, there has been an increased demand for engineered materials that are lightweight, stiff and tough. Interpenetrating Phase Composites (IPC), a family of bio-inspired materials, made of two or more phases where each phase forms a continuous 3-D network throughout the volume of the material could address some of these conflicting requirements. The specific IPC used in this work is formed by infusing uncured polymer syntactic foam which is prepared by dispersing hollow microballoons in epoxy into open-cell aluminum foam, and letting the syntactic foam to cure within the 3D metallic scaffold. The resultant is a composite (see, Fig. 1) of the syntactic foam and the aluminum foam which in their stand alone state are open cell foams themselves. In this work, the dynamic compressive responses of the syntactic foam and the hybrid syntactic foam-aluminum foam IPC are examined. The volume fraction of the hollow microballoons is varied and its effect on the dynamic compressive properties is studied experimentally.

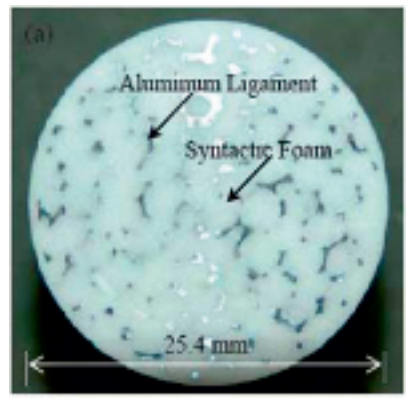

Fig. 1. Cross-section of IPC with 30\% volume fraction of SF

The dynamic compressive responses of the syntactic foam and the IPC are obtained by using a Split Hopkinson Pressure Bar (SHPB). The syntactic foam and IPC specimens of four different microballoon volume fractions - 10\%, 20\%, 30\% and 40\% are tested using the SHPB setup. All the dynamic tests are performed at a strain rate of approximately 1500 per second. The stress-strain responses and the energy absorption capacities are compared. For both syntactic foam and IPC samples, the yield stress decreases by $20 \mathrm{MPa}$ for every 10\% increase in the volume fraction. Also, the yield stresses of IPC are consistently higher than that of syntactic foam (SF) samples of corresponding volume fractions. This increase in the yield stress can be attributed to the fact that the aluminum ligaments in the IPC offer additional support to the structure. It is found that there is an increase of up to $14 \%$ in the yield stress of IPC when compared to the syntactic foam. A decreasing trend in the percentage increase of yield stress with increasing volume fractions in both the syntactic foam and IPC is also observed.

This is an Open Access article distributed under the terms of the Creative Commons Attribution-Noncommercial License 3.0, which permits unrestricted use, distribution, and reproduction in any noncommercial medium, provided the original work is properly cited. 

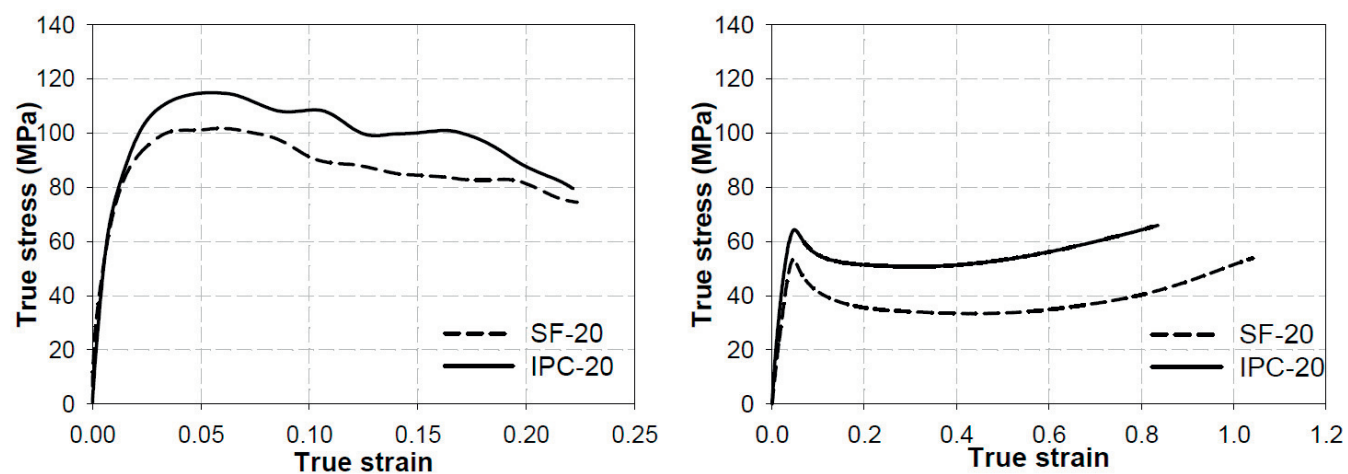

Fig. 2. Dynamic (left, strain-rate $\sim 1500 / \mathrm{sec}$ ) and static [3] (right, strain rate $\sim 0.001 / \mathrm{sec}$ ) stress - strain response of $20 \%$ volume fraction syntactic foam and IPC

The dynamic stress-strain responses are compared with their quasi-static counterparts. A representative comparison between static and dynamic responses of both SF and IPC is shown in Figure 2. Both SF and IPC samples are stiffer and have higher yield stress under dynamic loading conditions. In the static case, however, immediately following yielding there is a softening response signified by a visible drop in stress with increasing strain. In the dynamic case though, there is no evidence of such a softening behavior due to the highly transient nature of loading. The energy absorption during the dynamic loading of SF and IPC are found by calculating the area under the corresponding stress-strain curves. The IPC specimens consistently show higher energy absorption per unit volume when compared to the SF counterparts.

Finite element analysis using a unit cell approach is carried out to model measured dynamic responses of IPC. A tetrakaidecahedron (or a Kelvin cell) made of aluminum ligaments and filled with syntactic foam is modeled under elasto-plastic dynamic loading conditions. The measured high strain-rate stress-strain responses for SF and aluminum ligaments are used as inputs to the model. Infinite elements are adopted to mimic the dynamic response of a unit cell in an infinite IPC medium. Good agreement between the model and measurements are observed and a representative result for the case of 30\% volume fracture of microballoons is shown in Fig. 3.

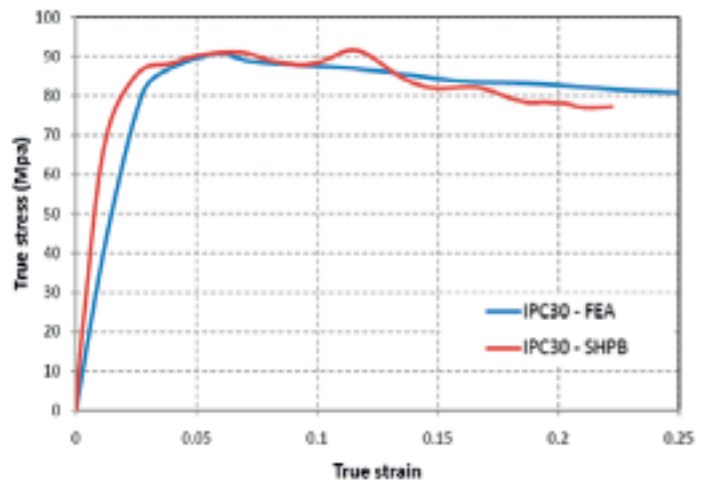

Fig. 3. Comparison between measured and computed stress-strain response of IPC with $30 \%$ volume fraction of microballoons 